\title{
Modeling and Analyzing Virtual Links Links Between the Sites of the Centers of the University of Mosul
}

\section{Basil Younis Al-Khayat}

Talal Fadel Hussein

talalmath@yahoo.com

College of Computer Science and Mathematics, University of Mosul

\section{Received on: 07/10/2012}

Accepted on: 30/01/2013

\section{ABSTRACT}

This paper deals with PageRanks which are used in the analysis of web links. It displays an introduction for the concept PageRanks and also some basic theoretical aspects related to it. It exposures three common methods for the purpose of calculating PageRanks: the direct method, the Markovian method and the power method. In order of fortification, these methods are applied on Internet sites being considered for the centers of the University of Mosul. Because the lack of realistic data, we dealt with hypothetical data for modeling and analysis of the links between sites centers affiliated to the University of Mosul. It is clear from the practical application of the three methods that they give consistent results.

Keywords: PageRanks, hypothetical links, University of Mosul.

$$
\begin{aligned}
& \text { نمذجة وتحليل وصلات روابط افتراضية بين مواقع المراكز التابعة لجامعة الموصل }
\end{aligned}
$$

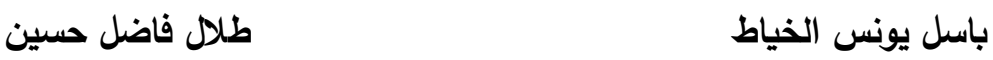

$$
\begin{aligned}
& \text { كلية علوم الحاسوب واليراضيات، جامعة الدوصل } \\
& \text { تاريخ قبول البحث: 2013/01/30 } \\
& \text { تاريخ استلام البحث: 2012/10/07 }
\end{aligned}
$$

رتبة بيج PageRank هي خوارزمية تحليل وصلة Link Analysis Algorithm، ولدت في جامعة

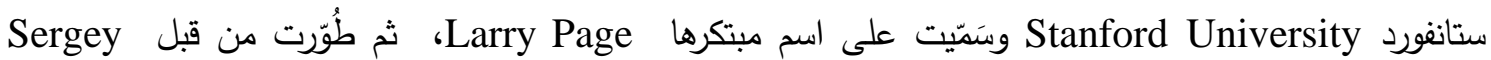

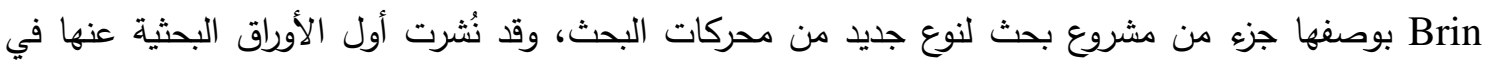

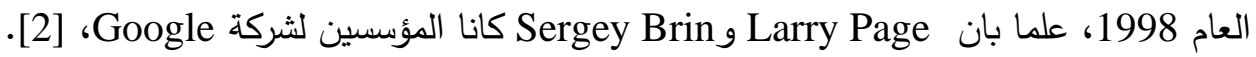

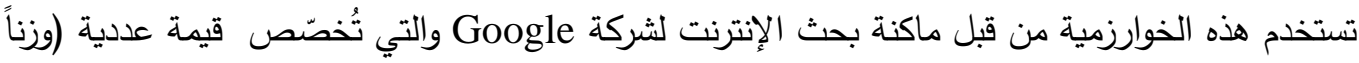

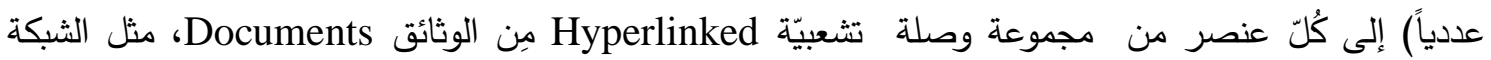
العالمية World Wide Web، لِغرض "قياس" الأهمية النسبية ضمن المجموعة. هذه الخوارزمية يمكن أن تُطبّق 
على أيّ مجموعة من الكيانات بالتقديرات والإشارات المتبادلة. إن الوزن العددي الذي يُخصّص إلى أيّ عنصر

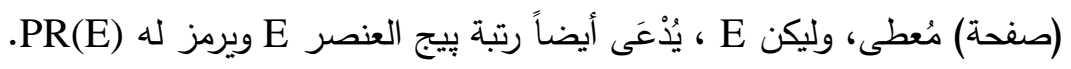
تحسب رتبة بيج عن طريق حل معادلة من 500 مليون متغير وأكثر من ملياري عبارة. وتعتمد رتبة بِيَج

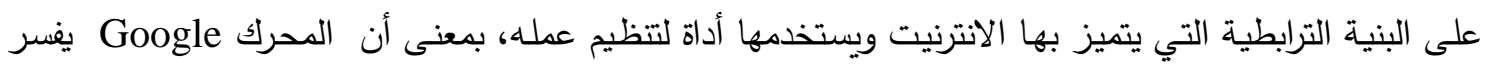

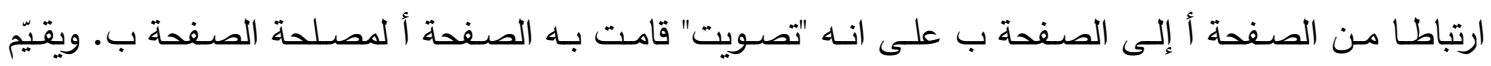
Google كانت الصفحات التي تصوّت "مهمة"، يعطيها تصويتها ذلك وزنا أكبر ، ويجعل الصفحات الأخرى التي تصوت لهات لهات لهات

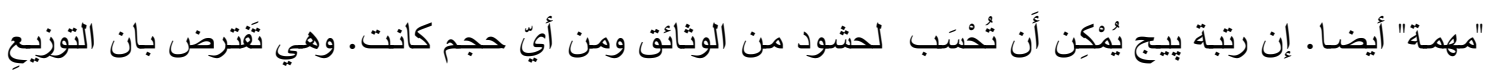
يُعسّم بانتظام بين كُلّ الوثائق في الحثد في بدِاية العملية الحسابية. تصف شركة Google رتبة بيهج بأنها تُكس رتبة بي-ج وجهة نظرها عن أهمية صفحات الويب باعتبار

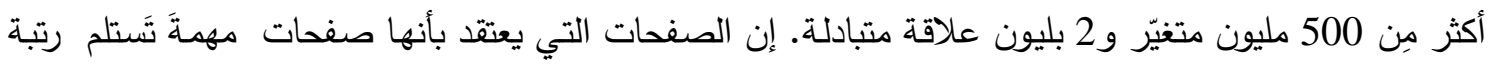

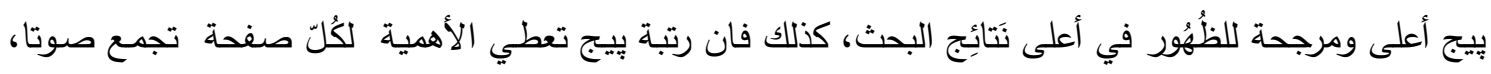

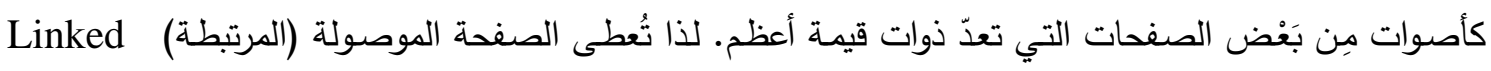

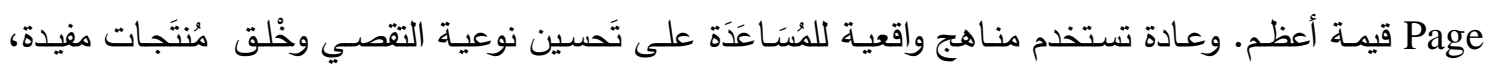

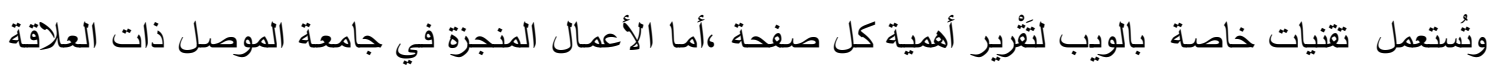
بالإنترنت فلم نجد سوى تقرير دبلوم في قسم علوم الحاسوب قدمه الباحث فتحي (2011)، إذ قام بتقديم أنموذج

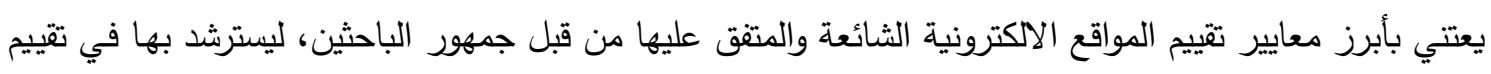
المواقع الاككترونية ويساعد في رفع مستويات الجودة، ويسهم في جعل إعداد المواقع وإدارتها عملاً علميًا وتعليميا.

\section{0 نظرية رتب بيج PageRank Theory}

في هذا المبحث نقدم خوارزمية مهمة جدا تستخدم من قبل جوجل في ترتيب صفحات الويب في الإنترنت في تصفح الإنترنت، عادة يستخدم المتصفحون محركات البحث للعثور على صفحات الويب ذات الصلة لتهن لتلبية

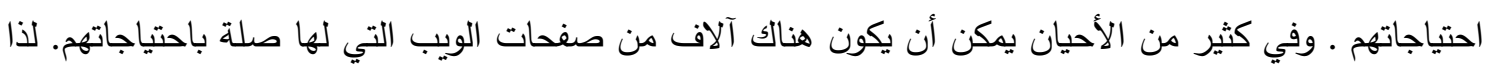
تبرز الحاجة لوضع قائمة للصفحات الويب وفق أهميتها وينبغي أيضا أن يتم تحديث هذه الأنيان القائمة بانتظام وبشكل

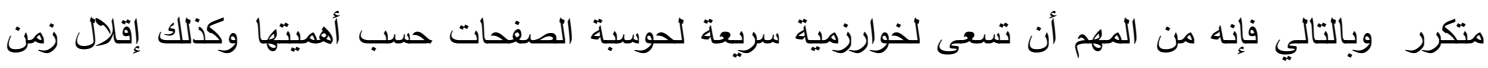

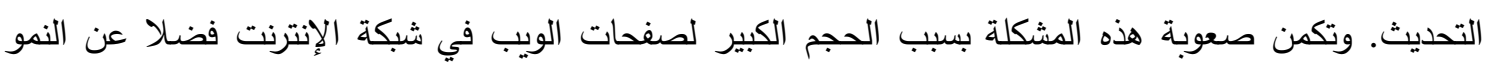

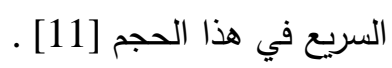

لقد ابتكر الباحثون [8] رتب بيج بوصفها القيم العددية التي تفيد في تحديد أهمية صفحات الويب وذلك

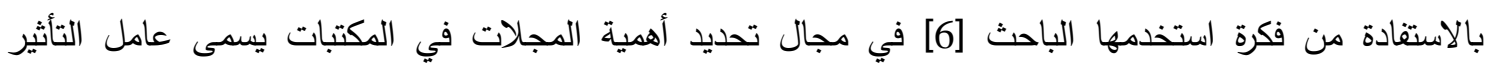
impact factor تعرّف رتب بيج على النحو الآتي: إذا كان N يمثل عدد صفحات الويب الكلية، فان مصفوفة الارتباط التشعبي تعرّف كما يأتي:

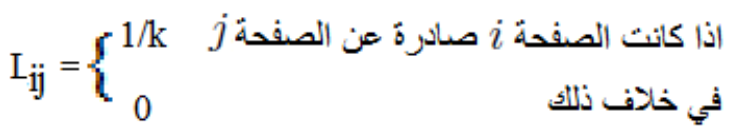


حيث إن k يمثل عدد جميع الصفحات الصادرة عن الصفحة ز. إن قيمة رتبة بيجج تعكس مقدار فرصة (حظ) الخادم (server) العشوائي الذي سيحط على الصفحة من

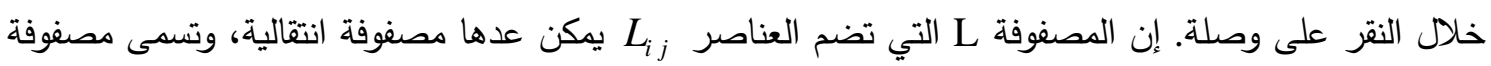
الوصلة التشعبية Hyperlink Matrix.

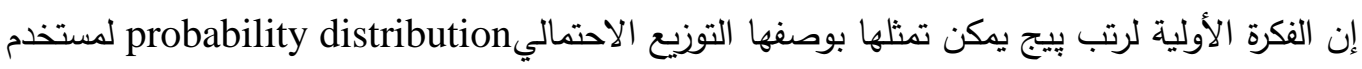

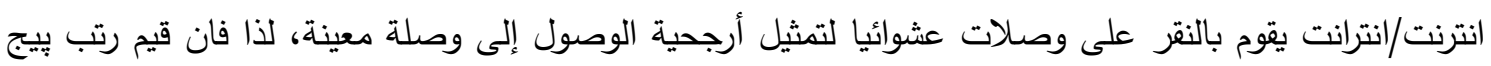

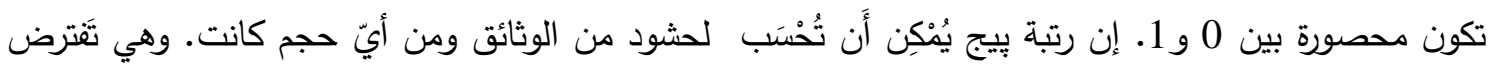

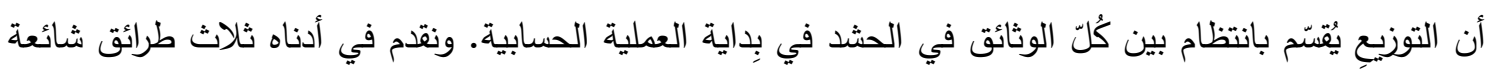

لحساب رتبة بي-ج.

1-2

لو كانت

تمثل الروابط الصادرة من

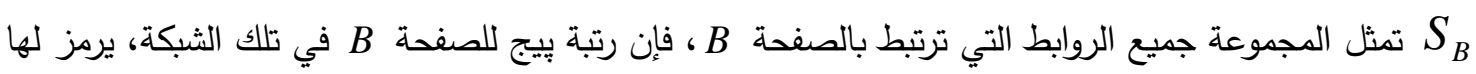
PR(B)

$P R(B)=\sum_{B \in S_{B}} \frac{P R(B)}{L(B)}$,

ولو كان damping factor يمثل عامل التثبيط (التخميد) da أحتمالية استمرار المستخدم في خطوة معينة) $P R(B)=\frac{1-d}{N}+d \sum_{B \in S_{B}} \frac{P R(B)}{L(B)}$, فيمكن إعادة كتابة المعادلة السابقة على النحو الآتي [4]: إذا أن 1> d> 0 . ونود الإشارة إلى أن القيمة المستخدمة عادة من عامل التثبيط هي حوالي

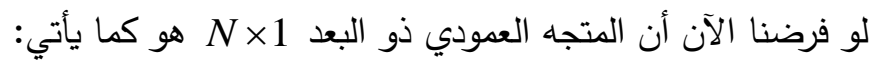

$R=\left[\begin{array}{c}P R\left(A_{1}\right) \\ \cdot \\ \cdot \\ \operatorname{PR}\left(A_{N}\right)\end{array}\right]$

يمثل متجه رتب بيج المطلوب إيجاده، إذ أن 1 إن . فيمكن تحويل المعادلة السابقة إلى معادلة

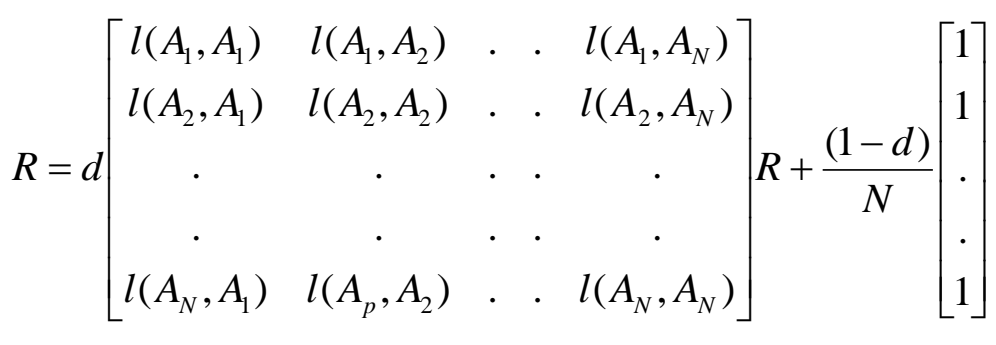
مصفوفات وكما يأتي : 
حيث أن adjacency function تمثل نسبة الانتقالات من $l\left(A_{i}, A_{j}\right)=0$

$\sum_{i=1}^{N} l\left(A_{i}, A_{j}\right)=1$ 1، أي تحقق القيد الآتي:

$R=d L R+\frac{(1-d)}{N}\left[\begin{array}{l}1 \\ 1 \\ \cdot \\ 1\end{array}\right]$

كما يمكن كتابة المعادلة (4) على النحو الآتي:

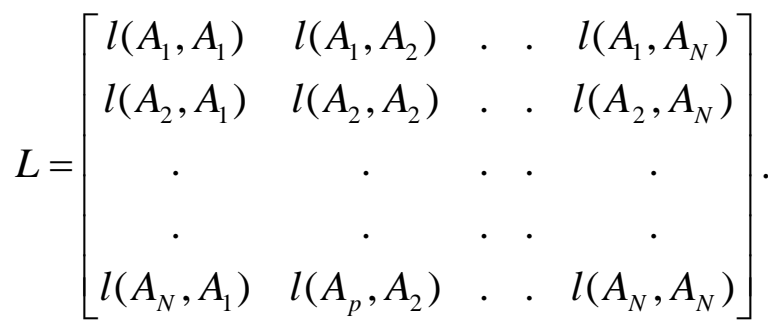

إذ أن

وتسمى المصفوفة Lبperlink matrix بمصفوفة الارتباط ألتشعبي ولحل المعادلة رقم (6) يمكن تحول هذه المسألة إلى مسألة متجه ذاتي eigenvector لغرض تسهيل المعالجة الرياضية وتقليل أخطاء التدوير ، [9].

2-2

Search Engines Markovian تستند هذه الطريقة على النمذجة الماركوفية لمحركات البحث

Modeling of

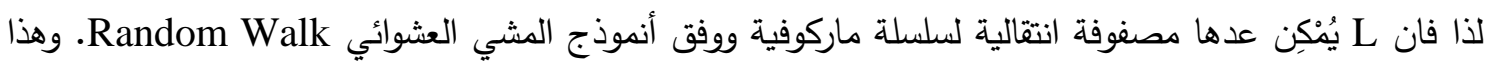
يعني أن الكُتصفح قد عُدّ ماشياً عشوائياً في حين أن صفحات الويب Webpage قد عُدّت حالات States لسلسلة ماركوفية. وبذلك يمكن نمذجة رتب بيجج وفق الأنموذج الماركوفي، حيث إن الحالات states تمثل الصفحات

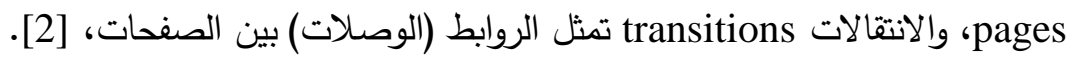

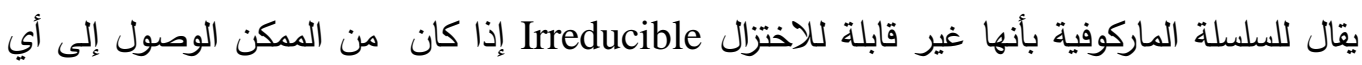

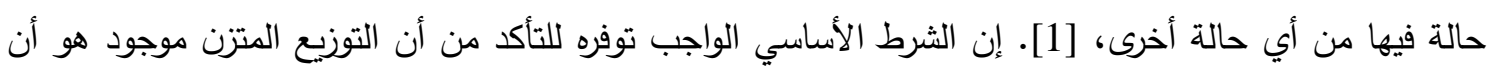
تكون السلسلة الماركوفية غير قابلة للاختزال Irreducible وغير دورية. وفي تطبيقات محركات البحث فربما

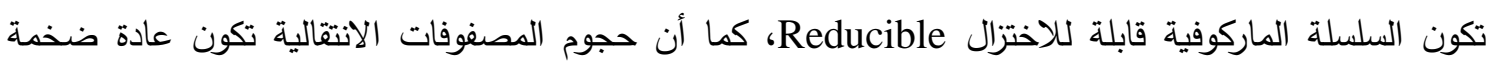
جدا. من ناحية أخرى فان الوقت المطلوب لحساب رتبة بِيج من قبل Google لا يتجاوز بضعة أيام. لذا فان الطريقة السابقة (الطريقة المباشرة) في حساب رتب بيجج (الاحتمالات المتزنة) ليست مرغوب فيها لكونهاب لكونها لا تلبي احتياجات هذه المسألة. لذا اهتم الباحثون في إيجاد بدائل، وهناك عدة طرائق متوافرة حاليا لمعالجة هذه المألكة. 
وبفرض أنَّ السلسلة الماركوفية غير قابلة للاختزال Irreducible وغير دورية، فان التوزيع المتزن يعرف رياضيا

على النحو الآتي، [1]

$\pi=\left(\pi_{0}, \pi_{1}, \ldots, \pi_{m}\right)=\lim _{n \rightarrow \infty} P^{n}$

حيث إن P تمثل مصفوفة الاحتمالات الانتقالية في الأنموذج الماركوفي. وبخصوص صفحات الويب فان التوزيع المتزن يكون موجودا وان $\pi_{i}$ تمثل نسبة الوقت الذي يقوم به الماشي العشوائي Random Walker (المتصفح (Surfer

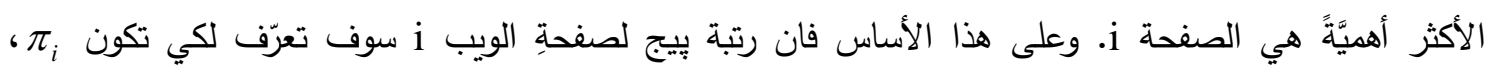
وسنطلق على هذه الطريقة في إيجاد رتبة بيج بالطريقة غير المباشرة.

3-2

تستخدم حاليا طريقة القوة power method على نطاق واسع لمعالجة مسألة رتبة بيجج. إن طريقة القوة

هي طريقة تكرارية iterative method لإيجاد اكبر قيمة ذاتية largest eigenvalue بالقيمة المطلقة وكذلك إيجاد المتجه الذاتي eigenvector المقابل، [7]. إن هذه الطريقة تقدم حلولا عملية لمسألة رتبة بيبج، إذ عندما يكون عدد صفحات الويب أكثر من 80 مليون صفحة وعند المعلمة تكرار فقط. للمعالجات الرياضية وتفاصيل طريقة القوة [5] ـ ونشير إلى أن طريقة القوة هي التي تعتمدها Google بهذا المجال. والخوارزمية الآتية تلخص طريقة القوة لإيجاد رتب بيجن. الخوارزمية (1): طريقة القوة لإيجاد رتب بيجج:

الخطوة (1): إدخال المصفوفة الانتقالية P. الخطوة (2): حساب المصفوفة Q من العلاقة الآتية: $Q=d\left[\begin{array}{ccccc}l\left(A_{1}, A_{1}\right) & l\left(A_{1}, A_{2}\right) & . & . & l\left(A_{1}, A_{N}\right) \\ l\left(A_{2}, A_{1}\right) & l\left(A_{2}, A_{2}\right) & . & . & l\left(A_{2}, A_{N}\right) \\ . & . & . & . & . \\ \cdot & \cdot & \cdot & . & . \\ l\left(A_{N}, A_{1}\right) & l\left(A_{N}, A_{2}\right) & . & . & l\left(A_{N}, A_{N}\right)\end{array}\right] P+\frac{(1-d)}{N}\left[\begin{array}{l}1 \\ 1 \\ \cdot \\ \cdot \\ 1\end{array}\right]$

الخطوة (3): إيجاد مقلوب transpose المصفوفة Q، أي 'Q الخطوة

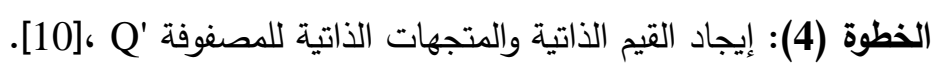
الخطوة (5): اخذ المتجه الذاتي الأول (الذي يقابل القيمة الذاتية الأولى والتي تساوي دائما 1). وبقسمة كل عنصة التصر

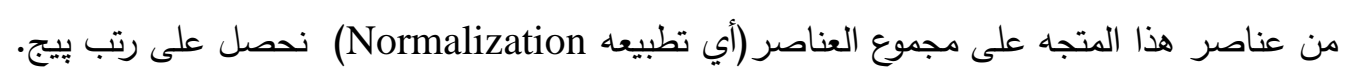
3- الروابط الافتراضية في جامعة الموصل وتحليل وصلاتها: يوجد في جامعة الموصل 7 مراكز تابعة لرئاسة الجامعة وهي:

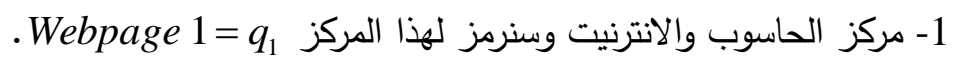

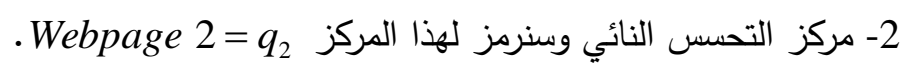


3- مركز طرائق التدريس وسنرمز لهذا المركز 3 .Webpage 3 .

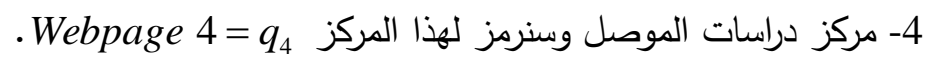

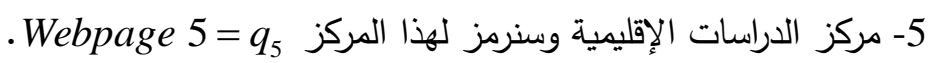

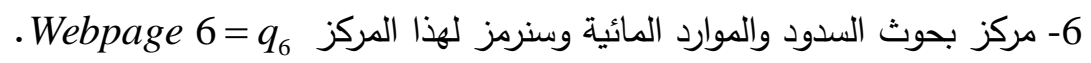

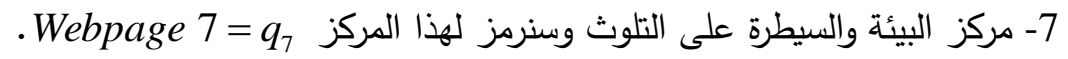

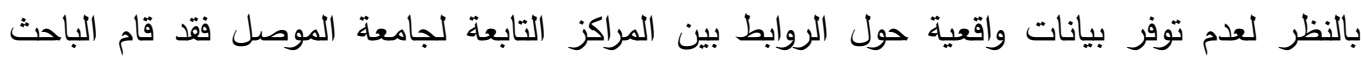

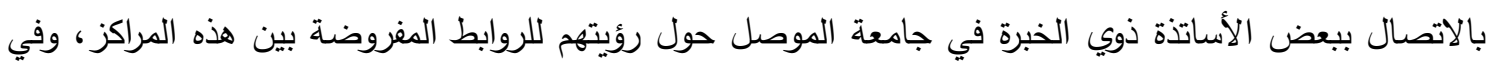
ضوء ذلك افترض أن الانتقالات هي كما مبينة في أدناه:

Webpage $1 \rightarrow 1,1 \rightarrow 2,1 \rightarrow 3,1 \rightarrow 4,1 \rightarrow 5,1 \rightarrow 6,1 \rightarrow 7$,

Webpage $2 \rightarrow 1,2 \rightarrow 2,2 \rightarrow 3,2 \rightarrow 6,2 \rightarrow 7$

Webpage $3 \rightarrow 1,3 \rightarrow 3$.

Webpage $4 \rightarrow 1,4 \rightarrow 4,4 \rightarrow 5$

Webpage $5 \rightarrow 1,5 \rightarrow 4,5 \rightarrow 5,5 \rightarrow 6,5 \rightarrow 7$

Webpage $6 \rightarrow 1,6 \rightarrow 2,6 \rightarrow 6,6 \rightarrow 7$,

Webpage $7 \rightarrow 1,7 \rightarrow 2,7 \rightarrow 6,, 7 \rightarrow 7$.

والثكل التالي يوضح المخطط الانتقالي بين صفحات الويب الكلية المفروضة الخاصة بالمراكز التابعة

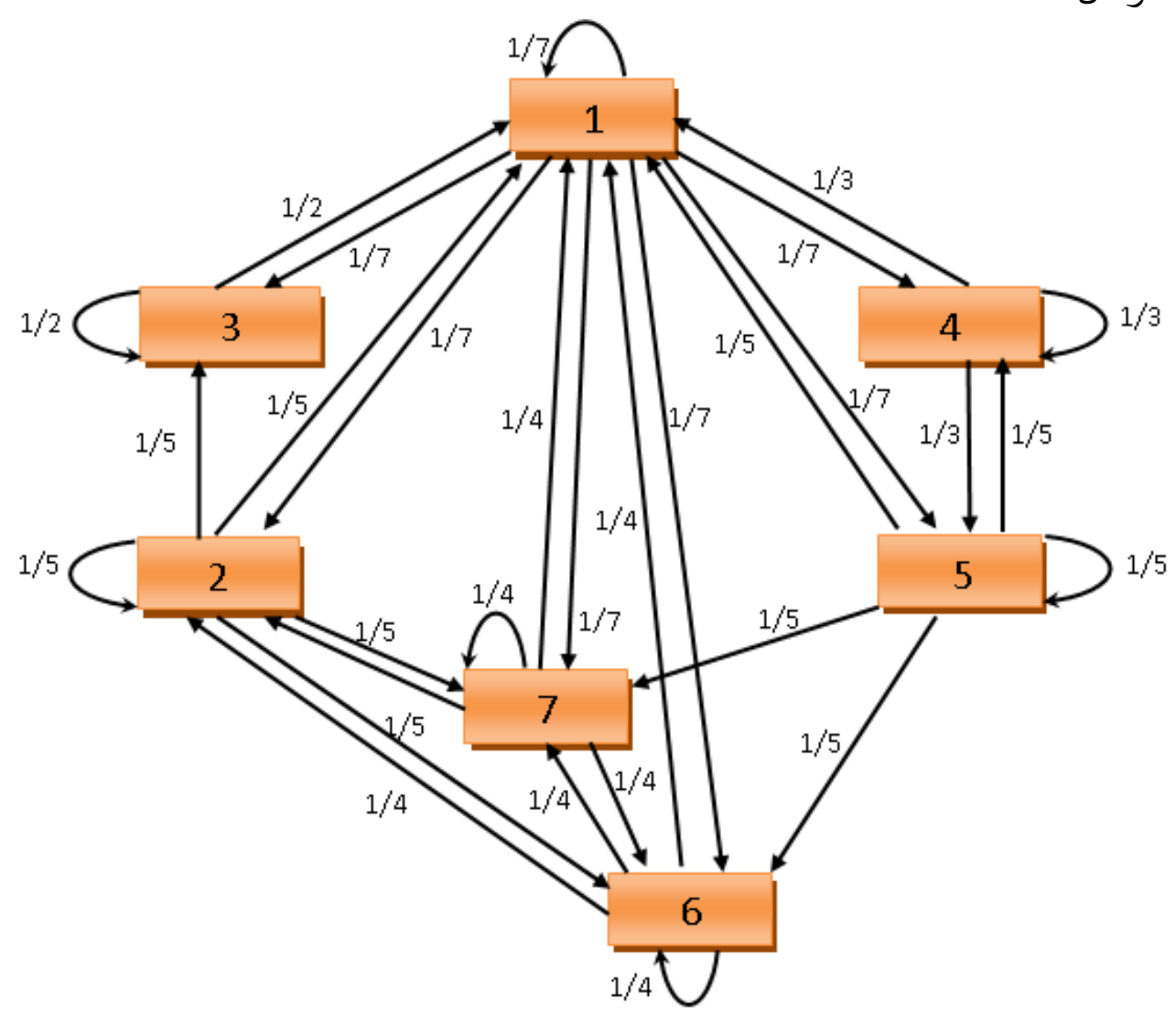

الثكل (1). المخطط الانتقالي بين صفحات الويب الكلية الخاصة بالمراكز التابعة لجامعة الموصل. 
والهدف من هذا البحث هو نمذجة وتحليل الوصلات بين صفحات الانترنت لهذه المراكز. ونقوم الآن إيجاد رتب بيجج باستخدام الطرائق الثلاث المذكورة سابقا: أولا: الطريقة المباشرة: بالتعويض في المعادلة (4)، نجد أن: $\left(\begin{array}{l}q_{1} \\ q_{2} \\ q_{3} \\ q_{4} \\ q_{5} \\ q_{6} \\ q_{7}\end{array}\right)=d\left(\begin{array}{ccccccc}1 / 7 & 1 / 7 & 1 / 7 & 1 / 7 & 1 / 7 & 1 / 7 & 1 / 7 \\ 1 / 5 & 1 / 5 & 1 / 5 & 0 & 0 & 1 / 5 & 1 / 5 \\ 1 / 2 & 0 & 1 / 2 & 0 & 0 & 0 & 0 \\ 1 / 3 & 0 & 0 & 1 / 3 & 1 / 3 & 0 & 0 \\ 1 / 5 & 0 & 0 & 1 / 5 & 1 / 5 & 1 / 5 & 1 / 5 \\ 1 / 4 & 1 / 4 & 0 & 0 & 0 & 1 / 4 & 1 / 4 \\ 1 / 4 & 1 / 4 & 0 & 0 & 0 & 1 / 4 & 1 / 4\end{array}\right)\left(\begin{array}{l}q_{1} \\ q_{2} \\ q_{3} \\ q_{4} \\ q_{5} \\ q_{6} \\ q_{7}\end{array}\right)+\frac{(1-d)}{7}\left(\begin{array}{l}1 \\ 1 \\ 1 \\ 1 \\ 1 \\ 1 \\ 1\end{array}\right)$.

$$
\left(\begin{array}{l}
q_{1} \\
q_{2} \\
q_{3} \\
q_{4} \\
q_{5} \\
q_{6} \\
q_{7}
\end{array}\right)\left(\begin{array}{c}
\frac{d}{7}\left(q_{1}+q_{2}+q_{3}+q_{4}+q_{5}+q_{6}+q_{7}\right) \\
\frac{0.85}{5}\left(q_{1}+q_{2}+q_{3}+q_{6}+q_{7}\right) \\
\frac{0.85}{2}\left(q_{1}+q_{3}\right) \\
\frac{0.85}{3}\left(q_{1}+q_{4}+q_{5}\right) \\
\frac{0.85}{5}\left(q_{1}+q_{4}+q_{5}+q_{6}+q_{7}\right) \\
\frac{0.85}{4}\left(q_{1}+q_{2}+q_{6}+q_{7}\right) \\
\frac{0.85}{4}\left(q_{1}+q_{2}+q_{6}+q_{7}\right)
\end{array}\right)+\left(\begin{array}{c}
\frac{1-d}{7} \\
\frac{1-d}{7} \\
\frac{1-d}{7} \\
\frac{1-d}{7} \\
\frac{1-d}{7} \\
\frac{1-d}{7} \\
\frac{0.15}{7}
\end{array}\right)
$$

$q_{1}=\frac{d}{7}\left(q_{1}+q_{2}+q_{3}+q_{4}+q_{5}+q_{6}+q_{7}\right)+\frac{1-d}{7}$

$q_{2}=\frac{d}{5}\left(q_{1}+q_{2}+q_{3}+q_{6}+q_{7}\right)+\frac{1-d}{7}$

$q_{3}=\frac{d}{2}\left(q_{1}+q_{3}\right)+\frac{1-d}{7}$

$q_{4}=\frac{d}{3}\left(q_{1}+q_{4}+q_{5}\right)+\frac{1-d}{7}$

$q_{5}=d\left(q_{1}+q_{4}+q_{5}+q_{6}+q_{7}\right)+\frac{1-d}{7}$ 


$$
\begin{aligned}
& q_{6}=\frac{d}{4}\left(q_{1}+q_{2}+q_{6}+q_{7}\right)+\frac{1-d}{7} \\
& q_{7}=\frac{d}{4}\left(q_{1}+q_{2}+q_{6}+q_{7}\right)+\frac{1-d}{7}
\end{aligned}
$$

$\frac{7-d}{7} q_{1}-\frac{d}{7}\left(q_{2}+q_{3}+q_{4}+q_{5}+q_{6}+q_{7}\right)=\frac{1-d}{7}$

$\frac{5-d}{5} q_{2}-\frac{d}{5}\left(q_{1}+q_{3}+q_{6}+q_{7}\right)=\frac{1-d}{7}$

$\frac{2-d}{2} q_{3}-\frac{d}{2} q_{1}=\frac{1-d}{7}$

$\frac{3-d}{3} q_{4}-\frac{d}{3}\left(q_{1}+q_{5}\right)=\frac{1-d}{7}$

$(1-d) q_{5}-d\left(q_{1}+q_{4}+q_{6}+q_{7}\right)=\frac{1-d}{7}$

$\frac{4-d}{4} q_{6}-\frac{d}{4}\left(q_{1}+q_{2}+q_{7}\right)=\frac{1-d}{7}$

$\frac{4-d}{4} q_{7}-\frac{d}{4}\left(q_{1}+q_{2}+q_{6}\right)=\frac{1-d}{7}$

ولحل المعادلات السبع الأخيرة، وبعد الاستفادة من القيد مصفوفات بالصيغة العامة المعروفة والتي هي بالثكل العام QX=B ، فيكون الحل العددي هو بالثكل

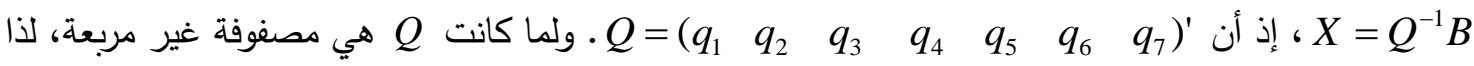

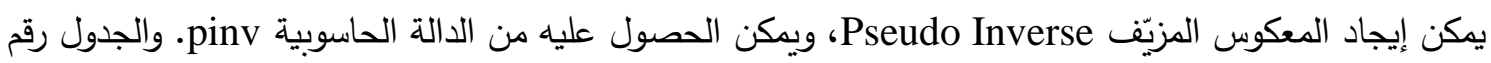
(1) يلخص النتائج التي تم الحصول عليها عند d=0.85:

الجدول (1). رتب بيج للروابط الافتراضية في جامعة الموصل وفق الطريقة المباشرة.

\begin{tabular}{|c|c|c|c|c|c|c|c|}
\hline Webpage & 1 & 2 & 3 & 4 & 5 & 6 & 7 \\
\hline وتبة بيجة & $\mathbf{- 0 . 0 3 2 7}$ & $\mathbf{0 . 0 3 7 8}$ & $\mathbf{0 . 0 1 3 1}$ & $-\mathbf{- 0 . 1 4 1 5}$ & $-\mathbf{- 0 . 4 0 0 9}$ & $\mathbf{0 . 0 3 9 2}$ & $\mathbf{0 . 0 3 9 2}$ \\
\hline
\end{tabular}

Webpage $6=$ Webpage $7>$ Webpage $2>$ Webpage $3>$ Webpage $1>$ Webpage $4>$ Webpage5

إذ أن القيم الأكبر تثير إلى صفحات الويب الأكثر أهمية. ثانيا: الطريقة غير المباشرة (استخدام الأنموذج الماركوفي): كما هو معروف فان العناصر الأساسية للأنموذج الماركوفي هي فضاء الحالة والمصفوفة الانتقالية، [1]. إن فضاء الحالة، والذي يمثل المجموعة التي تضم صفحات الويب الكلية الخاصة بالمراكز التابعة لجامعة الموصل، سيكون S=\{1,2,3,4,5,6,7). أما المصفوفة الانتقالية فستكون على النحو الآتي: 


$$
\begin{aligned}
& \begin{array}{lllllll}
1 & 2 & 3 & 4 & 5 & 6 & 7
\end{array}
\end{aligned}
$$

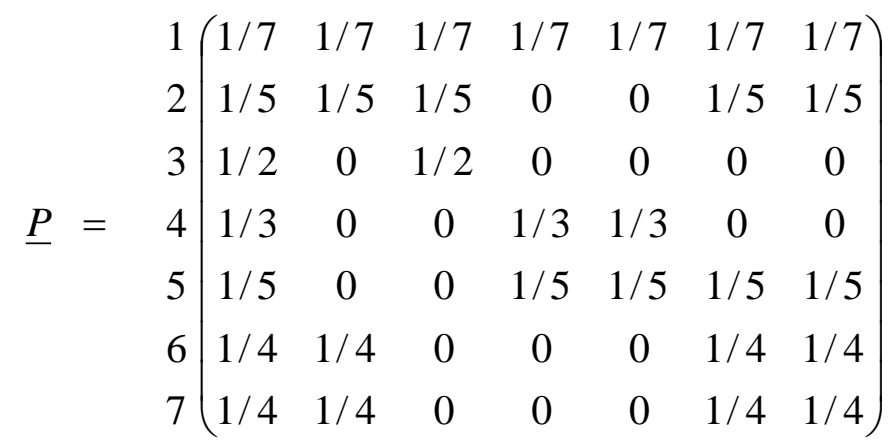

وكما هو واضح فان مجموع كل صف في المصفوفة السابقة يساوي 1.

إن رتبة بيجج تمثل التوزيع المتزن للأنموذج الماركوفي والذي يمكن الحصول عليه من حل المعادلة الآتية، [1]. $\underline{\pi}(I-P)=0$.

$$
I=\begin{aligned}
& 1 \\
& 2 \\
& 3 \\
& 4 \\
& 5 \\
& 6 \\
& 7 \\
& 7
\end{aligned}\left(\begin{array}{lllllll}
1 & 0 & 0 & 0 & 0 & 0 & 0 \\
0 & 1 & 0 & 0 & 0 & 0 & 0 \\
0 & 0 & 1 & 0 & 0 & 0 & 0 \\
0 & 0 & 0 & 1 & 0 & 0 & 0 \\
0 & 0 & 0 & 0 & 1 & 0 & 0 \\
0 & 0 & 0 & 0 & 0 & 1 & 0 \\
0 & 0 & 0 & 0 & 0 & 0 & 1
\end{array}\right)
$$

وان لصفحات الويب الكلية الخاصة بالمراكز التابعة لجامعة الموصل. وبحل المعادلة الأخيرة حصلنا على الحل الآتي:

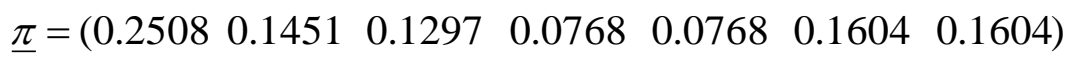

وباعتبار أن الاحتمالات المتزنة تمثل رُتب صفحات الويب السبع، فان الجدول رقم (2) يلخص النتائج التي تم الحصول عليها وفق هذه الطريقة:

\begin{tabular}{|c|c|c|c|c|c|c|c|}
\hline Webpage & 1 & 2 & 3 & 4 & 5 & 6 & 7 \\
\hline رتبة بِيج & 0.2508 & 0.1451 & 0.1297 & 0.0768 & 0.0768 & 0.1604 & 0.1604 \\
\hline
\end{tabular}

الجدول (2). رتب بيج للروابط الافتراضية في جامعة الموصل وفق الطريقة غير المباشرة.

ومن الجدول رقم (2) نستتج أن رُتب صفحات الويب ستكون (حسب أهميتها من حيث الاتصالات) على النحو

Webpage $1>$ Webpage6= Webpage7 $>$ Webpage $2>$ Webpage3 $>$ Webpage $4=$ Webpage 5

ثالثا: باستخدام طريقة القوة:

بفرض أن d=0.85 وباستخدام طريقة القوة تم إيجاد القيم الذاتية للمصفوفة الانتقالية P وكانت القيمة الذاتية الأولى 1، وكان المتجه الذاتي المقابل لهذه القيمة الذاتية: $\left[\begin{array}{lllllll}0.5958 & 0.3533 & 0.3242 & 0.2312 & 0.2312 & 0.3926 & 0.3926\end{array}\right]$ 
وبتحويل هذا المتجه الذاتي إلى متجه معياري وذلك بقسمته على مجموع عناصره (2.5207) تم الحصول على

$\left[\begin{array}{lllllll}0.2364 & 0.1401 & 0.1286 & 0.0917 & 0.0917 & 0.1557 & 0.1557\end{array}\right]$ المتجه الآتي: - 20 - 20 - 20 وهذا المتجه يمثل رتب بيج لصفحات الويب الكلية الخاصة بالمراكز التابعة لجامعة الموصل وهو موضح في

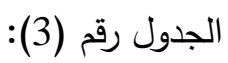

الجدول (3). رتب بيجج للروابط الافتراضية في جامعة الموصل وفق طريقة القوة.

\begin{tabular}{|c|c|c|c|c|c|c|c|}
\hline Webpage & 1 & 2 & 3 & 4 & 5 & 6 & 7 \\
\hline ونبة & 0.2364 & 0.1401 & 0.1286 & 0.0917 & 0.0917 & 0.1557 & 0.1557 \\
\hline
\end{tabular}

Webpage $1>$ Webpage $6=$ Webpage $7>$ Webpage $2>$ Webpage $3>$ Webpage $4=$ Webpage5

4- - الاستنتاجات والمناقثة:

الجدول رقم (4) يوضح نتائج رتب بيج للروابط الافتراضية في جامعة الموصل والتي تم الحصول عليها

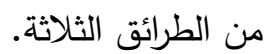
الجدول (4). رتب بيج للروابط الافتراضية والتي تم الحصول عليها من الطرائق الثلاثة.

\begin{tabular}{|c|c|c|c|c|c|c|c|}
\hline 7 & 6 & 5 & 4 & 3 & 2 & 1 & صفحة الويب حسب الأهمية \\
\hline 5 & 4 & 1 & 3 & 2 & 7 & 6 & الطريقة المباشرة \\
\hline 5 & 4 & 3 & 2 & 7 & 6 & 1 & الطريقة غير المباشرة \\
\hline 5 & 4 & 3 & 2 & 7 & 6 & 1 & طريقة القوة \\
\hline
\end{tabular}

وكما هو واضح فان نتائج طريقة القوة مطابقة تماما لنتائج الأنموذج الماركوفي. أما نتائج الطريقة المباشرة فتختلف قليلا عن الطريقتين السابقتين. ونشير إلى أن نتائج الطريقة المباشرة تعتمد على القيمة المختارة من المعلمة d فضلا عن أنها تتطلب حسابات كثيرة، لذا لا يوصى باستعمالها وبخاصة عندما يكون عدد الصفحات كبير

مما تقدم نجد أن رتب بِيج لصفحات الويب الكلية الخاصة بالمراكز التابعة لجامعة الموصل وفق الروابط المفروضة بين هذه المراكز ستكون على النحو الآتي: 1- مركز الحاسوب والانترنيت رتبته الأولى. 2- مركز بحوث السدود والموارد المائية رتبته الثانية. 3- مركز البيئة والسيطرة على التلوث رتبته الثالثة. 4- مركز التحس النائي رتبته الرابعة. 5- مركز طرائق التدريس رتبته الخامسة. 6- مركز دراسات الموصل رتبته السادسة. 7- 2 - مركز الدراسات الإقليمية رتبته السابعة. 


$$
\begin{aligned}
& \text { ألمصادر }
\end{aligned}
$$

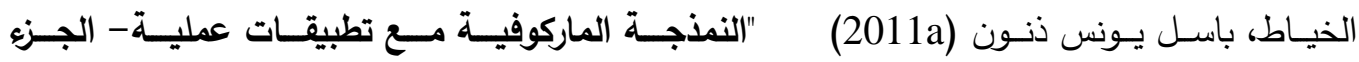

$$
\begin{aligned}
& \text { الأول "، دار ابن الأثير للطباعة والنشر ، جامعة الموصل. لـونس } \\
& \text { الخياط، باسل يونس ذنون (2011b) "النمذجة الماركوفية مع تطبيقات عملية- الجزء الثاني "، دار ابن }
\end{aligned}
$$

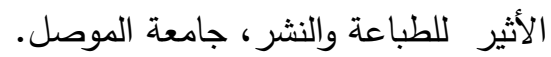

$$
\begin{aligned}
& \text { حسو، مهى عبد الرحمن و فتحي، فتحي غازي (2011) " دراسة معايير تثييم المواقع الالكترونية"، رسالة } \\
& \text { دبلوم عالي غير منشورة، كلية علوم الحاسوب والرياضيات، جامعة الموصل. }
\end{aligned}
$$

[4] Brin, S.; Page, L. (1998) "The anatomy of a large-scale hypertextual Web search engine". Computer Networks and ISDN Systems 30.

[5] Ching, Wai-Ki and Ng, Michael K. (2006) "Markov Chains: Models, Algorithms and Applications", Springer Science and Business Media, Inc.

[6] Garfield E (1972) "Citation Analysis as a Tool in Journal Evaluation", Science, 178.

[7] Golub G and van Loan C (1989) "Matrix Computations", The John Hopkins University Press.

[8] Page L, Brin S, Motwani R and Winograd T (1998), "The PageRank Citation Ranking: Bring Order to the Web", Technical Report, Stanford University.

[9] Ricardo, Henry (2010) "A Modern Introduction To Linear Algebra "(1st ed.), CRC Press.

[10] Ronald W. Shonkwiler (2009) "An Introduction to Parallel and Vector Scientific Computation", Georgia Institute of Technology pp. 206-230.

[11] Wenpu Xing, Ali Ghorbani (2004) " Weighted PageRank Algorithm", Proceedings of the Second Annual Conference on Communication Networks and Services Research (CNSR'04) IEEE computer society. 